Pacific Journal of Mathematic 


\title{
ON THE FRACTIONAL PARTS OF A SET OF POINTS II
}

\author{
R. J. CoOK
}

Heilbronn proved that for any $\varepsilon>0$ there exists a number $C(\varepsilon)$ such that for any real numbers $\theta$ and $N \geqslant 1$ there is an integer $n$ such that

$$
1 \leqslant n \leqslant N \quad \text { and } \quad\left\|n^{2} \theta\right\|<C(\varepsilon) N^{-1 / 2+\varepsilon}
$$

where $\|\alpha\|$ denotes the difference between $\alpha$ and the nearest integer, taken positively. The method depends on Weyl's estimates for trigonometric sums. The result was generalized by Davenport who obtained analogous results for polynomials which have no constant term.

The object here is to obtain a result for simultaneous approximations to quadratic polynomials $f_{1}, \cdots f_{R}$ having no constant term:

For any $\varepsilon>0$ there is a number $C=C(\varepsilon, R)$ such that for any $N \geqslant 1$ there is an integer $n$ such that

$$
\begin{array}{r}
1 \leqslant n \leqslant N \quad \text { and } \quad\left\|f_{i}(n)\right\|<C N^{-1 / g(R)+\varepsilon} \\
\text { for } i=1, \cdots, R,
\end{array}
$$

where $g(1)=3$ and $g(R)=4 g(R-1)+4 R+2$ for $R \geqslant 2$.

1. Introduction. In 1948 Heilbronn [4] proved the result stated above on the distribution of the sequence $n^{2} \theta(\bmod 1)$. This was generalized to polynomials which have no constant term by Davenport [2].

THEOREM. Let $\varepsilon>0$ and let $R$ be a positive integer. Then there is a number $C=C(\varepsilon, R)$ such that for any quadratic polynomials $f_{1}, \cdots, f_{R}$ having no constant term, and for any $N \geqslant 1$, there is an integer $n$ such that

$$
1 \leqslant n \leqslant N \quad \text { and }
$$

$$
\begin{array}{r}
\left\|f_{i}(n)\right\|<C N^{-1 / g(R)+\varepsilon} \\
\text { for } i=1, \cdots, R,
\end{array}
$$

where

$$
g(1)=3 \text { and } g(R)=4 g(R-1)+4 R+2 \text { for } \quad R \geqq 2,
$$

the result being uniform in $f_{1}, \cdots, f_{R}$.

It can be readily verified by induction that an explicit formula for $g(R)$ is

$$
18 g(R)=29 \cdot 4^{R}-24 R-20, \quad \text { for } R \geqq 2 \text {. }
$$


2. Preliminaries to the proof. The case $R=1$ was proved by Davenport [2]. The theorem will be proved by induction on $R$, so we suppose the theorem is true for $R-1$. $\varepsilon$ denotes a small positive number and $r(\varepsilon)$ denotes a multiple of $\varepsilon$ depending only on $R$, note that $r(\varepsilon)$ differs in its various occurrences. We may suppose that $N>N_{0}(\varepsilon, R) . \quad F \ll G$ means that $|F|<C G$ where $C$ depends at most on $\varepsilon$ and $R . e(z)=\exp (2 \pi i z)$.

Lemma 1 (Vinogradov). Let $\Delta$ satisfy $0<\Delta<1 / 2$ and let $a$ be a positive integer. Then there exists a function $\psi(z)$, periodic with period 1, which satisfies

$$
\psi(z)=0 \quad \text { for }\|z\|>\Delta
$$

and

$$
\psi(z)=\sum_{m=-\infty}^{\infty} a_{m} e(m z)
$$

where the $a_{m}$ are real numbers, $a_{0}=\Delta, a_{m}=a_{-m}, m=1,2, \cdots$, and

$$
\left|a_{m}\right|<A \min \left(\Delta, m^{-a-1} \Delta^{-a}\right), \quad m \neq 0,
$$

where $A$ depends only on a.

Proof. This is a particular case of Lemma 12 of Chapter 1 of Vinogradov [5].

Lemma 2 (Weyl). Let $A$ and $P$ be real numbers, $P \geqslant 1$. Let $\alpha=a q^{-1}+\beta$ where $(a, q)=1, q \geqslant 1$ and $|\beta| \leqq q^{-2}$. Then

$$
\left|\sum_{A \leqq n \leqq A+P} e\left(\alpha n^{2}+\alpha_{1} n\right)\right|^{2} \ll P^{\varepsilon}\left(q^{-1} P+1\right)(P+q \log q) \text {. }
$$

Proof. See, for example, Lemma 1 of Davenport [1].

Let

$$
f_{i}(n)=\theta_{i} n^{2}+\phi_{i} n, \quad i=1, \cdots, R .
$$

We choose a positive number $\delta$ so that there is no integer $n$ with

$$
1 \leqslant n \leqslant N \quad \text { and } \quad\left\|f_{i}(n)\right\| \leqslant N^{-\delta}, \quad i=1, \cdots, R .
$$

We may suppose that $\delta<1 / g(R)$. We take $\Delta=N^{-\delta}$ and $a=\left[2 \varepsilon^{-1}\right]+1$ in Lemma 1. Then

$$
\sum_{n=1}^{N} \prod_{i=1}^{R} \psi\left(f_{i}(n)\right)=0
$$

so

$$
N^{1-R \hat{o}}+\Sigma^{*} a_{m_{1}} \cdots a_{m_{R}} T(\boldsymbol{m})=0
$$


where $\Sigma^{*}$ denotes a summation over $-\infty<m_{1}<\infty, \cdots,-\infty<m_{R}<\infty$, $\boldsymbol{m}=\left(m_{1}, \cdots, m_{R}\right) \neq 0$,

$$
T(\boldsymbol{m})=\sum_{n=1}^{N} e\left(\boldsymbol{m} \cdot \boldsymbol{\theta} n^{2}+\boldsymbol{m} \cdot \boldsymbol{\phi} n\right),
$$

$$
\boldsymbol{m} \cdot \boldsymbol{\theta}=\sum_{i=1}^{R} m_{i} \theta_{i} \quad \text { and } \quad \boldsymbol{m} \cdot \boldsymbol{\phi}=\sum_{i=1}^{R} m_{i} \phi_{i} .
$$

Summing over terms in the region $\left|m_{1}\right|>N^{\delta+\varepsilon}$ we have

$$
\begin{aligned}
\sum\left|a_{m_{1}} \cdots a_{m_{R}} T(\boldsymbol{m})\right| & \ll N \sum N^{a \delta} m_{1}^{-a-1} \\
& \ll N^{1-a \varepsilon}
\end{aligned}
$$

by Lemma 1, and similarly for other regions $\left|m_{i}\right|>N^{\delta+\varepsilon}$. Thus

$$
\begin{aligned}
1 & \ll N^{-1+R \delta} \Sigma^{\prime}\left|a_{m_{1}} \cdots a_{m_{R}} T(\boldsymbol{m})\right| \\
& \ll N^{-1} \Sigma^{\prime}|T(\boldsymbol{m})|
\end{aligned}
$$

where $\Sigma^{\prime}$ denotes a summation over $\max \left|m_{i}\right| \leqslant N^{\delta+\varepsilon}, \boldsymbol{m} \neq \mathbf{0}$. Taking the square of this inequality and applying Cauchy's inequality we have

$$
1 \ll N^{-2+R \delta+R \varepsilon} S
$$

where

$$
S=\Sigma^{\prime}|T(m)|^{2}
$$

We now proceed to estimate $S$. Let $Q=N^{A}, T=N^{B}$ where $A$ and $B$ will be chosen later. By Dirichlet's theorem on Diophantine approximation, see Theorem 185 of Hardy and Wright [3], for each $\boldsymbol{m}$ there exist integers $a, b, q$ and $t$ such that

$$
\begin{array}{ll}
\boldsymbol{m} \cdot \boldsymbol{\theta}=a q^{-1}+\alpha \text { with }(a, q)=1, \quad 1 \leqslant q \leqslant Q, & q|\alpha| \leqslant Q^{-1} \\
\boldsymbol{m} \cdot \boldsymbol{\phi}=b t^{-1}+\beta \text { with }(b, t)=1, \quad 1 \leqslant t \leqslant T, \quad t|\beta| \leqslant T^{-1} .
\end{array}
$$

3. The induction step. For any $m$ in the sum for $S$ we have

$$
\max \left|m_{i}\right| \leqslant N^{\delta+\varepsilon} \text {. }
$$

Since $\boldsymbol{m} \neq \mathbf{0}$ and $|T(-\boldsymbol{m})|=|T(\boldsymbol{m})|$ we may suppose that $m_{R}>\mathbf{0}$. We take

$$
\begin{gathered}
\sigma=2 g(R-1) \delta+4 g(R-1) \varepsilon, \\
A=\frac{3}{2}+(2 g(R-1)+1) \delta+(4 g(R-1)+3) \varepsilon,
\end{gathered}
$$

and 


$$
B=\frac{1}{2}+2 \varepsilon
$$

Applying the case $R-1$ of the theorem to the polynomials

$$
f_{i}^{*}(n)=m_{R} q^{2} t^{2} \theta_{i} n^{2}+q t \phi_{i} n, i=1, \cdots, R-1,
$$

we see that there is an integer $x$ such that

$$
\begin{array}{r}
1 \leqslant x \leqslant N^{\sigma} \quad \text { and } \quad\left\|f_{i}^{*}(x)\right\| \ll N^{-\sigma / g(R-1)+\varepsilon}, \\
\quad i=1, \cdots, R-1 .
\end{array}
$$

Suppose that $q<N^{1 / 2-\sigma-\delta-4 \varepsilon}$. Taking $y=m_{R} q t x$ we have $1 \leqq y \leqq N$ and for $i=1, \cdots, R-1$

$$
\begin{aligned}
\left\|f_{i}(y)\right\| & =\left\|m_{R}^{2} q^{2} t^{2} \theta_{i} x^{2}+m_{R} q t \phi_{i} x\right\| \\
& \leqslant\left|m_{R}\right|\left\|f_{i}^{*}(x)\right\| \leqslant N^{-\delta},
\end{aligned}
$$

by (16), (17), and (21). Also

$$
\begin{aligned}
\left\|f_{R}(y)\right\|= & \left\|m_{R}^{2} q^{2} t^{2} \theta_{R} x^{2}+m_{R} q t \phi_{R} x\right\| \\
\leqslant & \left\|m_{R} q^{2} t^{2} x^{2} \boldsymbol{m} \cdot \boldsymbol{\theta}\right\|+\left\|\sum m_{i}\left(m_{R} q^{2} t^{2} \theta_{i} x^{2}+q t \phi_{i} x\right)\right\| \\
& +\left\|\sum m_{i} q t \phi_{i} x+m_{R} q t \phi_{R} x\right\| \\
\leqslant & \left|m_{R} q t^{2} x^{2}\right|\|q \boldsymbol{m} \cdot \boldsymbol{\theta}\|+\sum\left|m_{i}\right|\left\|f_{i}^{*}(x)\right\| \\
& +|x q|\|t \boldsymbol{m} \cdot \boldsymbol{\phi}\| \\
\leqslant & N^{-\delta}
\end{aligned}
$$

by $(14)-(21)$, where the summations are over $i=1, \cdots, R-1$.

This contradicts the assumption that there were no integer solutions of (8). Therefore $q \geqslant N^{1 / 2-\sigma-\delta-4 \varepsilon}$.

4. Completion of the proof of the theorem. From (6) we have

$$
|T(\boldsymbol{m})|^{2} \ll q^{-1} N^{2+\varepsilon}+q N^{\varepsilon}+N^{1+\varepsilon} \text {. }
$$

For $N^{1 / 2-\sigma-\delta-4 \varepsilon} \leqslant q \leqslant N$ we have

$$
|T(\boldsymbol{m})|^{2} \ll q^{-1} N^{2+\varepsilon} \ll N^{1+1 / 2+(2 g(R-1)+1) \delta+r(\varepsilon)} \text {. }
$$

Summing over $O\left(N^{R(\delta+\varepsilon)}\right)$ such $\boldsymbol{m}$ we have a contribution $S_{1}$ to $S$ where

$$
S_{1} \ll N^{1+1 / 2+(2 g(R-1)+R+1) \dot{o}+r(\varepsilon)} .
$$

For $N \leqslant q \leqslant M=N^{A}$ we have

$$
|T(\boldsymbol{m})|^{2} \ll q N^{\varepsilon} \ll N^{1+1 / 2+(2 g(R-1)+1) \delta+r(\varepsilon)} .
$$

Summing over $O\left(N^{R(\delta+\varepsilon)}\right)$ such $\boldsymbol{m}$ we have a contribution $S_{2}$ to $S$ where 
(28)

$$
S_{2} \ll N^{1+1 / 2+(2 g(R-1)+R+1) \delta+r(\varepsilon)} .
$$

Therefore, from (12), we have

$$
1 \ll N^{-1 / 2+(2 g(R-1)+2 R+1) \delta+r(\varepsilon)} .
$$

Hence

$$
-\varepsilon<-\frac{1}{2}+(2 g(R-1)+2 R+1) \delta+r(\varepsilon)
$$

so

$$
\delta>1 / g(R)-r(\varepsilon)
$$

and the theorem is proved.

\section{REFERENCES}

1. H. Davenport, Analytic Methods for Diophantine Equations and Diophantine Inequalities, Ann Arbor, Michigan, 1962.

2. - On a theorem of Heilbronn, Quart. J. Math. Oxford, (2), 18 (1967), 339344.

3. G. H. Hardy and E. M. Wright, An Introduction to the Theory of Numbers, 4th ed., Oxford, 1965.

4. H. Heilbronn, On the distribution of the sequence $n^{2} \theta(\bmod 1)$, Quart. J. Math. Oxford, 19 (1948), 249-256.

5. I. M. Vinogradov, The Method of Trigonometrical Sums in the Theory of Numbers, (Translated by K. F. Roth and Anne Davenport), Interscience Publishers, 1954.

Received December 14, 1971.

UNIVERSity COLLEGE, CARDIFF 



\section{PACIFIC JOURNAL OF MATHEMATICS}

EDITORS

\author{
H. SAMELSON \\ Stanford University \\ Stanford, California 94305 \\ C. R. HOBBY \\ University of Washington \\ Seattle, Washington 98105
}

\author{
J. DUGUNDJI \\ Department of Mathematics \\ University of Southern California \\ Los Angeles, California 90007 \\ RICHARD ARENS \\ University of California \\ Los Angeles, California 90024
}

\section{ASSOCIATE EDITORS}

E. F. BECKENBACH

B. H. NeUManN

F. WOLF

K. YosHIDA

\section{SUPPORTING INSTITUTIONS}

UNIVERSITY OF BRITISH COLUMBIA

CALIFORNIA INSTITUTE OF TECHNOLOGY

UNIVERSITY OF CALIFORNIA

MONTANA STATE UNIVERSITY

UNIVERSITY OF NEVADA

NEW MEXICO STATE UNIVERSITY

OREGON STATE UNIVERSITY

UNIVERSITY OF OREGON

OSAKA UNIVERSITY
UNIVERSITY OF SOUTHERN CALIFORNIA

STANFORD UNIVERSITY

UNIVERSITY OF TOKYO

UNIVERSITY OF UTAH

WASHINGTON STATE UNIVERSITY UNIVERSITY OF WASHINGTON

AMERICAN MATHEMATICAL SOCIETY NAVAL WEAPONS CENTER 


\section{Pacific Journal of Mathematics}

\section{Vol. 45, No. $1 \quad$ September, 1973}

William George Bade, Complementation problems for the Baire classes .......... 1

Ian Douglas Brown, Representation of finitely generated nilpotent groups ........ 13

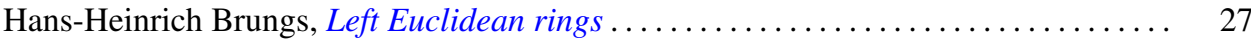

Victor P. Camillo and John Cozzens, A theorem on Noetherian hereditary rings ..... 35

James Cecil Cantrell, Codimension one embeddings of manifolds with locally flat

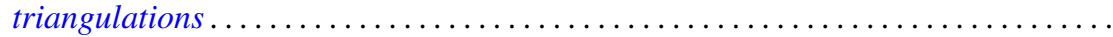

L. Carlitz, Enumeration of up-down permutations by number of rises . . . . . . . . . .

Thomas Ashland Chapman, Surgery and handle straightening in Hilbert cube

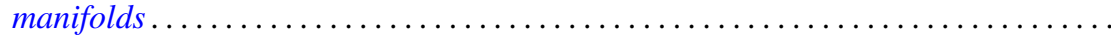

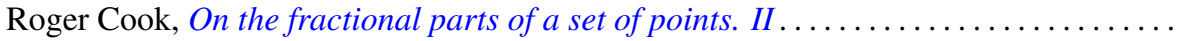

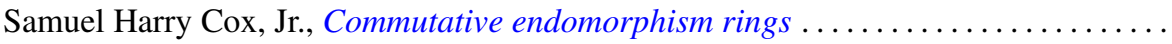

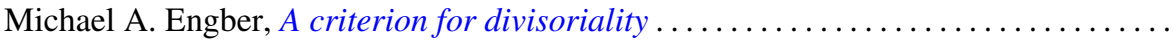

Carl Clifton Faith, When are proper cyclics injective . . . . . . . . . . . . . . 97

David Finkel, Local control and factorization of the focal subgroup . . . . . . . . . 113

Theodore William Gamelin and John Brady Garnett, Bounded approximation by

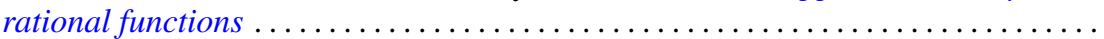

Kazimierz Goebel, On the minimal displacement of points under Lipschitzian

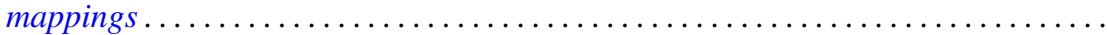

Frederick Paul Greenleaf and Martin Allen Moskowitz, Cyclic vectors for representations associated with positive definite measures: nonseparable

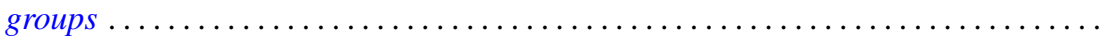

Thomas Guy Hallam and Nelson Onuchic, Asymptotic relations between perturbed linear systems of ordinary differential equations .

David Kent Harrison and Hoyt D. Warner, Infinite primes of fields and completions. .

James Michael Hornell, Divisorial complete intersections . ......

Jan W. Jaworowski, Equivariant extensions of maps ..............

John Jobe, Dendrites, dimension, and the inverse arc function .. .

Gerald William Johnson and David Lee Skoug, Feynman integrals of non-factorable

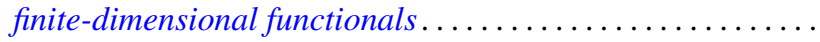

Dong S. Kim, A boundary for the algebras of bounded holomorphic functions ...... 269

Abel Klein, Renormalized products of the generalized free field and its derivatives ... 275

Joseph Michael Lambert, Simultaneous approximation and interpolation in $L_{1}$ and

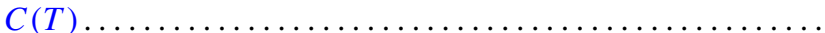

Kelly Denis McKennon, Multipliers of type $(p, p)$ and multipliers of the group

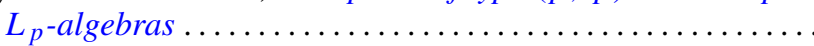

William Charles Nemitz and Thomas Paul Whaley, Varieties of implicative

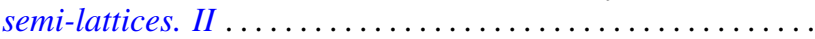

Donald Steven Passman, Some isolated subsets of infinite solvable

Norma Mary Piacun and Li Pi Su, Wallman compactifications on E-completely

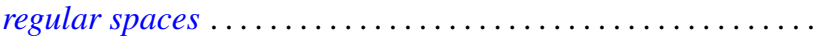

Jack Ray Porter and Charles I. Votaw, $S(\alpha)$ spaces and regular Hausdorff extensions....

Gary Sampson, Two-sided $L_{p}$ estimates of convolution transforms .

Ralph Edwin Showalter, Equations with operators forming a rig
Raymond Earl Smithson, Fixed points in partially ordered sets .

Victor Snaith and John James Ucci, Three remarks on symmetric products and

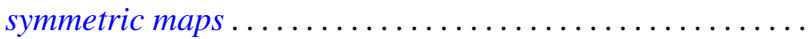

